\title{
Ovarian, Fallopian Tube, and Primary Peritoneal Carcinoma pT1b TNM Finding v8
}

National Cancer Institute

\section{Source}

National Cancer Institute. Ovarian, Fallopian Tube, and Primary Peritoneal Carcinoma

pT 1b TNM Finding v8. NCI Thesaurus. Code C139944.

Ovarian, fallopian tube, or primary peritoneal carcinoma with tumor limited to one or both ovaries (capsules intact) or fallopian tubes; no tumor on ovarian or fallopian tube surface; no malignant cells in ascites or peritoneal washings. (from AJCC 8th Ed.) 